\title{
Carnets
}

Revue électronique d'études françaises de l'APEF

Première Série - 4 | 2012

(Res)sources de l'extravagance

\section{Pourquoi les morts sont-ils si extravagants?}

\section{João Domingues}

\section{(2) OpenEdition \\ Journals}

Édition électronique

URL : http://journals.openedition.org/carnets/6627

DOI : 10.4000/carnets.6627

ISSN : 1646-7698

Éditeur

APEF

Édition imprimée

Date de publication : 1 janvier 2012

Pagination : 47-62

Référence électronique

João Domingues, « Pourquoi les morts sont-ils si extravagants? », Carnets [En ligne], Première Série 4 | 2012, mis en ligne le 20 juin 2018, consulté le 01 mai 2019. URL : http://journals.openedition.org/ carnets/6627 ; DOI : 10.4000/carnets.6627

Carnets est mis à disposition selon les termes de la licence Creative Commons - Atribution - Pas d'utilisation commerciale 4.0 International. 


\title{
POURQUOI LES MORTS SONT-ILS SI EXTRAVAGANTS?
}

\author{
JOÃO DOMINGUES \\ Centre de Littérature Portugaise \\ Universidade de Coimbra \\ jcosta@fl.uc.pt
}

\section{Résumé}

Dans le genre du dialogue, le "dialogue des morts" est un sous-genre mineur: relevant souvent de la littérature anonyme et clandestine, il est à vrai dire son avatar extravagant, plein de bizarreries et de paradoxes. S'autorisant de Lucien de Samosate et de ses Dialogues des morts, ces textes mettent en valeur une tradition satirique qui, au moins depuis Aristophane, permet, par exemple, d'invectiver publiquement les plus grands de ce monde. De cette typologie émerge un monde à l'envers, souvent entendu comme carnavalesque, en tout cas propre à une littérature "hors cadre" et aux sujets surprenants. L'analyse de quelques unes de ces "descentes littéraires" chez les morts fera ressortir la richesse, la profondeur et l'originalité thématique et stylistique de ce sous-genre cultivé depuis au moins deux millénaires et qui se nourrit de l'extravagance même dans toute son ampleur.

\begin{abstract}
"Why the dead are so extravagant?" In the Dialogue genre, "dialogue of the dead" is a minor subgenre, often originating in the anonymous and clandestine literature, is indeed, an extravagant avatar, full of oddities and paradoxes. From this typology there emerges a world upside down, often perceived as a Carnival; in any case, specific to a literature, defying classification and thriving on unexpected topics. The analysis of some of these literary "descent to the dead" texts will highlight the richness, depth, thematic and stylistic originality of this sub-genre produced from at least two millennia ago that still lives through the extravagance of all its magnitude.
\end{abstract}

Mots-clés: absurde, déraison, extravagance, paradoxe, ridicule

Keywords: absurd, extravagance, nonsense, paradox, ridiculous 
Dans ses Dialogues des morts, Lucien de Samosate avait "marié" le genre du dialogue - qui était sérieux - à celui de la comédie; c'est la première extravagance du genre. Puis, attribuer aux morts la dure tâche de parler de la vie des hommes et de leur société leur sujet préféré -, faire parler ces Ombres conversant dans les Champs-Elysées ou dans les Enfers, ce qui revient au même, est d'une extravagance à toute épreuve, d'autant plus que ces morts n'abdiquent pas de leur omniscience ou presque, et encore moins de leur grande liberté de morts: car rien ne peut plus les arrêter, même pas la mort. Ce sont des extra-vagants par excellence, ce qui leur confère beaucoup d'avantages. Ils n'ont pourtant pas surmonté leurs propres défauts, qu'ils n'ont d'ailleurs aucune peine à exposer, ce qui rend ces juges de l'humanité presque toujours de bonne humeur, aussi bien lucides que ridicules.

Le dialogue était à l'époque classique en France un genre assez ouvert et non codifié, propre à toute sorte de libertinage de la pensée, à cette pensée étincelante et diverse, et c'est là justement toute sa richesse.

\section{Fontenelle}

L'imitation la plus célèbre des Dialogues des morts de Lucien, qui furent traduits et publiés en français par Nicolas Perrot d'Ablancourt en 1654, est sans aucun doute les Nouveaux dialogues de morts de Fontenelle, parus à Paris en $1683^{1}$. L'auteur ne dément pas ce qu'il doit à l'auteur grec car il le reconnaît et le justifie dès le début de l'ouvrage dans sa lettre "À Lucien aux Champs Elisiens", qui constitue l'épitre dédicatoire de l'ouvrage. L'idée est la même - faire parler les morts, et le dessein aussi - la critique typiquement "lucianesque"; la seule différence par rapport à son mentor serait l'introduction de nouveaux sujets: “j'ay quelque lieu d'espérer”, dit Fontenelle, "que le dessein qui est de vous, fera passer les choses qui sont de moy" (Fontenelle, 1971: 97). Un autre trait le distingue encore de Lucien: au lieu de faire parler les dieux et les morts, Fontenelle préfère limiter ces conversations aux seuls morts, car, dit-il, "l'Histoire me fournissoit assez de veritables Morts, et d'Aventures veritables, pour me dispenser d'emprunter aucun secours de la Fiction" (Ibidem: 99-100). Le choix est donc fait: la vraisemblance historique, malgré tout, au détriment de la fantaisie mythologique. Ce choix ne relève pourtant que de l'attention que l'homme du monde et très raisonnable Fontenelle voue au goût de son siècle; par contre, pour les sujets comme pour la critique, sous-jacente ou explicite, il n'y aura fort heureusement presque pas de limites: "comme les Morts ont bien de l'esprit, ils doivent voir

\footnotetext{
${ }^{1}$ L'édition que nous utilisons ici est l'édition critique avec une introduction et des notes par Jean d'Agen, Librairie Marcel Didier, Paris 1971.

Dans toutes les citations, nous avons respecté la graphie de l'époque dans les titres des dialogues et dans les extraits.
} 
bientôt le bout de toutes les matières" (Ibidem: 99), observe-t-il encore en ton provocateur, ce qui annonce, dès lors, un esprit libre et beaucoup d'extravagance.

L'expression claire et simple mais toujours insinuante dans chaque dialogue, par laquelle l'auteur fait preuve d'un esprit vif et profondément critique, présente au lecteur d'innombrables situations ou faits historiques dont le but n'est que la remise en cause, voire même le refus de toute sorte de pensée ou de coutume prétendue normale ou incontestable par le sens commun, souvent dans des domaines assez intouchables pour l'époque comme la religion catholique et son église. Par conséquent, dès sa parution, les Nouveaux dialogues des morts ont fait l'objet de critiques très violentes, de Baltus, père jésuite, comme de Le Tellier, confesseur du roi à l'époque, entre autres. Pris pour un auteur dangereux, le jeune Fontenelle eut la chance d'avoir, pour le protéger, non seulement la célébrité de ses oncles, les frères Corneille, mais aussi Tournemine, un père jésuite qui le connaissait depuis son jeune âge au Collège de Rouen et qui a pris sans délai sa défense dans le Journal de Trévoux'. Le marquis d'Argenson lui-même est intervenu pour que le bras séculier n'entamât pas de poursuite contre le jeune écrivain (cf.: Fayol, 1961:22).

Dans les Nouveaux dialogues des morts, reines et courtisanes, astrologues et conquérants se rencontrent et entament la conversation sur leurs vies et leurs idées en toute liberté et sans aucune forme de contrainte; mais, outre leurs grandes différences sociales, ils proviennent de lieux très éloignés et même de temps si séparés qu'apparemment ils n'auraient rien en commun, rien à se dire, rien à faire ensemble. Le résultat de la conversation est, lui aussi, souvent paradoxal: en effet, s'ils s'entendent plutôt bien au cours de leurs rencontres, le lecteur, lui, finit par comprendre que les Sages et les Grands de ce monde ne sont pas comme l'Histoire nous les a transmis; que les vrais sages ne sont jamais ceux que l'on croyait; que Socrate ne fut qu'un galant et Alexandre-le-Grand un fléau pour l'Humanité, alors qu'une simple courtisane comme Phryné avait agi toute sa vie avec une morale irréprochable et un grand amour pour l'Humanité; que l'Histoire est pleine d'éloges immérités comme de vitupérations injustes; enfin, que la raison et la vérité ne sont jamais là où l'ont croyait pouvoir les atteindre. Voilà des révélations dérangeantes que font ces morts sur l'histoire du monde et des hommes de tous les temps.

Ces morts sont de vraies "mémoires vivantes"; le grand savoir qu'elles portent leur permet en effet d'engager la conversation avec l'interlocuteur le plus inattendu; mais en même temps elles se manifestent assez ignorantes pour que la conversation garde tout son naturel. Par conséquent, le lecteur n'aura jamais la sensation que ces morts ne font que débiter des choses préalablement connues de tous. Aussi le changement opéré chez certains personnages est-il tellement inattendu qu'ils scandalisent parfois leurs interlocuteurs

\footnotetext{
${ }^{2}$ Ce journal, créé par les jésuites et imprimé à Trévoux, était destiné à combattre les idées de "l'école philosophique"; Tournemine fut un de ses directeurs.
} 
et ne laissent pas de surprendre le lecteur le plus averti. C'est d'ailleurs par ce genre d'extravagance que "le dialogue des morts" crée une ambiance de surprise permanente qui nourrit son style typiquement provocateur indépendamment du sujet abordé.

Dans l'utilisation de l'Histoire, Fontenelle a exclusivement recours à des personnages historiques. Pourquoi? "Parce qu'il s'agit de connaître et de comprendre l'homme. Or la vérité de l'homme est à découvrir dans le comportement des hommes, elle n'est pas le produit d'un système", explique Dagen dans son introduction (1971: 21); mais c'est presque la seule stratégie de vraisemblance chez Fontenelle. À partir de là, soit pour la mise ensemble des personnages soit pour le déroulement de leurs discours, c'est la provocation, le paradoxe, voire même l'absurde qui conduit chaque dialogue à une pensée dernière, ou à la manifestation d'une vérité souvent contraire à celle qu'a diffusée l'Histoire; une extravagance, certes, mais très souvent au service d'une vérité plus humaine et plus profonde. Ainsi Caton d'Utique avoue qu'il s'est suicidé, non pas par noblesse d'esprit comme l'histoire nous l'a fait croire, mais simplement parce qu'il n'a pas eu le choix; l'empereur Adrien raconte qu'il n'est pas mort joyeusement en récitant des vers badins, mais bien rompu de douleur causée par son hydropisie, celle-là oui, bien réelle, si douloureuse et fatale; enfin, Alexandre-Le-Grand reconnaît, devant la courtisane Phryné, que "courir toûjours, sans sçavoir où, et de prendre toûjours des Villes, sans sçavoir pourquoy, et d'exécuter toûjours, sans avoir aucun dessein" ${ }^{3}$, comme il a fait pendant toute sa vie, c'est le propre d'un insensé et non pas d'un héros. Par conséquent, le vrai Alexandre aurait donc été un pauvre homme fou d'ambition et non pas un héros! Ce n'est peut-être pas vraisemblable, mais la vérité ne l'est pas souvent non plus: tantôt les mêmes causes produisent des effets contraires, tantôt des causes différentes produisent le même effet. La condition humaine est souvent déterminée par une logique qui nous dépasse; et vraiment,

quoy qu'on fasse dans le monde, on ne sçait ce que l'on fait; et (...) on doit trembler mesme dans les affaires où l'on se conduit bien, et craindre de n'avoir pas fait quelque faute qui eust été necessaire. Tout est incertain ${ }^{4}$;

on aurait envie de compléter: tout est extravagant!

Un discours désabusé sur l'homme. Mais pourquoi les morts sont-ils si extravagants? Pour mieux détromper les vivants de leurs illusions et les rendre plus raisonnables. Toute l'extravagance des morts comme tous les ridicules des hommes ainsi dénoncés ne servent qu'à présenter les "petites vérités" d'une Histoire vraie de l'Humanité qui reste à écrire.

\footnotetext{
${ }^{3}$ Fontenelle (1971), "Alexandre, Phriné": 119-120.

4 Ibidem, "Athenaïs et Icasie": 150.
} 
C'est à cette fin précisément que le langage de ces morts paraît n'avoir pas de limites que celles de l'imagination extravagante par nature; or il n'y a pas plus grande extravagance que de franchir les limites de la mort, pour jouir d'une liberté sans bornes, d'expression et de fait, précisément celle dont jouissent les morts. Dépasser ces limites, jouir de cette extravagance, veut dire ne plus avoir affaire aux contraintes physiques, d'ordre matériel ou temporel. Les morts jouissent d'une liberté suprême. Même leurs corps ne leur sont plus à charge, ni leurs âmes, puisqu'ils les ont déjà rendues. Ils sont donc l'extravagance même. Pour eux, le temps n'est plus: des morts anciens conversent tranquillement avec des morts modernes sans se rendre compte qu'il y a entre eux un abîme de plusieurs siècles; mais qu'est-ce pour eux si d'un seul coup d'œil il leur est possible d'appréhender toute l'Histoire? Voilà ce qui permet et à quoi aboutit ce dépassement des limites temporels par ces morts omniscients ou presque. Aussi le message sort-il des limites de l'histoire, devenant par là atemporel. De même, le dépassement de tous les tabous historiques ou de civilisation incarne la subversion de l'histoire, de toute chronologie comme de toute hiérarchie. Et pourtant, quand les morts réfléchissent sur la condition des vivants, ils voient que la nature commande sur nous en maîtresse toute puissante, nous imposant en tout certaines limites, en connaissances comme en espérances, en jouissances comme en désirs, en capacité d'atteindre la vérité comme en espérance de l'atteindre, et que, dans ces conditions, il serait même permis aux hommes de se demander jusqu'à quel point il serait bon d'être fou. En tout cela, très paradoxalement, les morts sont encore les plus raisonnables et les moins extravagants.

L'Extravagance n'est qu'un masque du très raisonnable Fontenelle. Chez Fontenelle, ce sont les sources mêmes de ses Nouveaux dialogues des morts qui, d'un éclectisme assez large, il est vrai, et dépassant souvent le recours raisonnable à l'histoire, nous semblent assez extravagantes. Fontenelle se plaît à chercher dans les recoins les moins connus de l'histoire ses personnages qu'il exhibe comme s'ils étaient les plus dignes représentants de l'humana gens. En plus de l'extravagance du choix des personnages, la formation des "couples" qui dialoguent n'arrête pas d'étonner le lecteur. Des gens venus de mondes différents, ayant vécu à des époques séparées par des siècles d'histoire, conversent tranquillement comme ils s'insultent ou parlent d'amour: extravagance profonde, mais pas si folle que ça, car ces sujets, étant morts, ne sont-ils pas de tous les temps et de tous les âges? L'homme, n'est-il pas toujours le même, inchangeable dans son essence? II n'y a que les modes et les conditions qui changent au travers des temps car elles sont éphémères; mais l'essentiel ne change jamais!

En bon émule de Lucien, Fontenelle moralise tous ses dialogues. Cependant, il s'érige plutôt en moraliste qu'en moralisateur: il ne choisit pas que de bons exemples; il choque, il scandalise le lecteur avec ses choix, d'autant plus que souvent, face aux 
exemples des plus célèbres héros de l'Histoire, il se trouve que ce sont les "mauvais" qui nous semblent avoir raison! Or cette extravagance est peut-être la meilleure voie pour démontrer la vanité de l'héroïsme et de la renommée face à la destinée humaine, égale pour tous, grands et petits, célèbres ou inconnus.

Comble de l'extravagance - mais c'est là aussi qu'il lève un peu le voile - chez Fontenelle, que de soumettre "Les dialogues des morts" au "Jugement de Pluton", alors qu'il avait affirmé auparavant qu'il se passerait bien des dieux ridicules, puisque seule la vie des hommes l'intéressait. Le fait est que, à cause de ces "dialogues", les Enfers vivaient dans le plus grand désordre, avec les Courtisanes qui se jetaient dans le quartier des Héros et les rois qui faisaient la cour aux Savants; et Pluton, lui-même mécontent du traitement que l'auteur lui fait subir, s'exclame:

Morts. Où diable l'Autheur des Dialogues a-t-il pris que j'étais usé? Je luy feray voir qu'il n'en est rien. Que tout l'Enfer soit témoin de ma vengeance, et que le bruit en aille jusqu'à la Boutique de Blageart ${ }^{6}$.

Entre les vivants et les morts, les mondes se (con)fondent. Les critiques que certains morts exposent devant le roi des Enfers, mécontents qu'ils sont du traitement que leur ont infligé leurs partenaires, ainsi que les verdicts que profère Pluton à l'encontre de tous ces médisants, s'adressent tous, on l'aura compris, plutôt aux lecteurs vivants qu'aux personnages de ces mêmes dialogues. Lucien a appris à Fontenelle, son Copiste, à "ne respecter rien de tout ce que le monde respecte. Lucien attaque tout ce qu'il connoist de plus grand et de plus élevé; le Copiste en fait autant" (Ibidem: 426-427). C'est la règle du jeu. "Dans les Nouveaux Dialogues, c'est une règle infaillible que vous trouverez toûjours tout renversé. Du moment que vous voyez ensemble un Sage et un Fou, assurez-vous que le Fou sera bien au dessus du Sage" (Ibidem.: 429). C'est la voix ironique de l'auteur, en contrebas, faisant des Enfers un théâtre de comédie, un monde à l'envers.

Mais l'incongruité ne s'arrête pas là, car Pluton venait à peine de donner son dernier verdict pour apaiser les morts lorsque Mercure entra en plein milieu de l'assemblée pour annoncer qu'il venait d'arriver de dessus la Terre et que les Vivants lui avaient confié une Lettre pour les Morts. Et le lecteur d'apprendre que, si les morts se plaignent d'être maltraités ou mal compris dans ces dialogues, les Vivants viennent à leur tour les informer que, ayant lu ces mêmes textes, ils les ont pris au sérieux! Puis Caron annonce encore une nouveauté: une seconde partie des dialogues! Et il s'ensuit après une seconde partie du

\footnotetext{
${ }^{5}$ Face à de nombreuses plaintes de certains morts aux enfers, qui n'ont pas été bien ménagés dans ces dialogues ou qui ont simplement été oubliés pas eux. Pluton a été obligé de leur livrer publiquement certains verdicts condamnant ces "scandales" et ordonnant qu'ils ne se répètent plus.

${ }^{6}$ Fontenelle (1971), "Jugement de Pluton sur les Dialogues de morts": 421.
} 
"Jugement de Pluton"; enfin, l'ouvrage se termine en vraie comédie burlesque puisque, à l'annonce de nouveaux dialogues de ce genre, la folie s'empare de la foule des morts.

Or les morts, dans toute leur extravagance, "sont gens de grande réflexion tant à cause de leur expérience que de leur loisir"7, remarquait déjà Fontenelle dans son introduction. Et en effet, si les personnages et leurs façons sont extravagants, les pensées qu'ils expriment ne le sont point; tout au contraire, elles relèvent plutôt d'un regard très objectif et sans aucune forme d'ambigüité, d'excès ou d'illusion. Dans les N.d.m. de Fontenelle comme dans certains de leurs émules, la philosophie qui s'est égarée du droit chemin doit revenir à sa première et vraie fonction. Pour ce faire, elle doit adopter le scepticisme: une approche critique du "spectacle du monde", devant lequel la raison doit commencer par vérifier et avouer ses propres impuissances et, par conséquent, accepter certains renoncements. Les sciences sont, en général, fallacieuses ou vaines; en tout cas elles n'expliquent nullement le système du monde. Renonçant donc à vouloir harmoniser l'ordre connu par Dieu et le désordre que l'homme subit sur terre, aucune théodicée ne l'intéresse; la Nature lui suffit. Au lieu de vouloir comprendre le monde, mieux vaut consentir à l'habiter et y vivre.

Dans une perspective imbue d'humanisme, il n'est plus essentiel de comprendre la structure du monde; c'est l'homme qu'il nous faut comprendre, l'homme qui se révèle dans l'Histoire, par la vie politique et sociale comme par l'amour: I'homme esclave des passions, enchaîné dans la société par ses valeurs comme par ses préjugés, pourvu d'une raison inefficace mais désireux de lucidité. S'exercer au doute serait par conséquent le meilleur exercice de l'intelligence. En effet, évitant de se duper par le préjugé, par la tradition, la raison démontre son pouvoir tout en détruisant ses propres mirages. La reconnaissance de ses impuissances est une partie essentielle à la rigueur qui est la rançon de sa propre liberté.

Lucide et profonde, cette critique est pourtant joyeuse, voire triomphante; un scepticisme qui se manifeste, comme nous l'avons vu, par l'humour; une raison joyeuse qui, au lieu de s'indigner face aux absurdités et au mal de ce monde, préfère les prendre avec les pinces de l'ironie, ou en rire tout simplement. Aussi, tout en philosophant sur des sujets les plus graves, la verve et la hardiesse extravagante de nos morts lui confèrent-elles une allure d'humoriste de bonne humeur.

De l'extravagance au doute perpétuel. L'option pour la folie, comme chez Guillaume de Cabestan $^{8}$, est encore une autre extravagance; mais peut-on choisir vraiment? C'est l'ensemble des folies de chacun qui composent la société: préjugés, passions et folies

\footnotetext{
${ }^{7}$ Ibidem, "Épître à Lucien”.

8 Ibidem, "Guillaume de Cabestan, Albert-Frideric de Brandebourg".
} 
finissent par modeler l'univers de l'homme. Quant à la raison, si elle est, elle se trouve cachée dans la Nature qui garde pour elle-même la vérité; en effet, "l'ordre que la Nature a voulu établir dans l'univers va toujours son train"'. Elle a l'air bien constant et même ce que l'on nomme hasard n'est "qu'un ordre que l'on ne connaît point". Et "quand nous découvrons le peu d'importance de ce qui nous touche, nous arrachons à la Nature son secret; on devient trop sage, on n'est pas assez homme"10. Or ce qui vient d'être énoncé n'est plus une extravagance: c'est bien le nouveau sens de la relativité de tout, y compris de tout ce qui touche à l'homme. Sans angoisse, certes, mais aussi sans le culte d'une raison conquérante, ce relativisme montre que seule l'existence a du prix et du sens. Pour le reste, tout laisse à croire que, dans les N.d.m., on n'est pas loin de l'époché qui mène à cette "suspension de l'esprit qui est le but de l'œuvre de l'auteur, et le point de la félicité tout ensemble" (1716: 23).

Enfin, chez Fontenelle, l'extravagance des personnages, du sujet de leurs conversations ou des conclusions qu'on peut en tirer est à l'image de la pensée même de l'auteur: derrière toute la panoplie d'exagérations extravagantes, la pensée de Fontenelle surgit dans les méandres même de chaque dialogue et s'annonce très raisonnable et surtout désabusée. En effet, si Lucien se servait des dieux et de la mythologie pour montrer les torts humains, Fontenelle, lui, se sert des morts historiques et de leurs extravagances pour en faire montrer combien est ridicule le comportement humain lorsqu'il est dominé par l'ambition de la gloire, du pouvoir ou des richesses, par les croyances et les fausses vertus de toute sorte. Curcius a donné sa vie pour la patrie; mais ont l'aurait détrompé avant en lui disant "il est de vostre devoir de vous jetter dans cet abîme, mais soyez sûr que personne ne parlera jamais de vostre action"11, et il aurait préféré fuir. Lucrèce, ce modèle de vertu, avoue qu'elle a agi par orgueil (Ibidem: 351), et Artemise s'est immolée par ostentation ${ }^{12}$. Où est donc le courage? Où est la vertu? Puis, dans une espèce de philosophie de l'absurde avant la lettre, ces morts nous montrent que tout cela est inutile, puisque tout marche inexorablement vers la même fin, le néant: pour Bérénice, il est absolument indifférent qu'on ait donné son nom à toute une constellation, car elle n'en est pas moins morte ${ }^{13}$; David Riccio n'a même pas senti que la reine Marie Stuart lui avait octroyé le plus grand honneur de reposer pour toujours dans la tombe des rois d'Écosse ${ }^{14}$; enfin, Jeanne de Naples ne put avoir du grand astrologue que la seule prédiction possible: "vous êtes morte et vous le serez toujours"15.

\footnotetext{
9 Ibidem, "Lucrece, Barbe Plomberge": 352.

10 Ibidem, "Parmenisque, Theocrite de Chio": 288.

11 Ibidem, "Lucrèce, Barbe Plomberge": 351.

12 Ibidem, "Artemise, Raimond Lulle": 313.

13 Ibidem, "Bérénice, Cosme II. De Médicis": 194.

14 Ibidem, "Marie Stuart, David Riccio": 373.

15 Ibidem, "Jeanne de Naples, Anselme": 237.
} 


\section{Les imitations de Lucien}

Cependant, dans le genre du dialogue des morts, Fontenelle est loin d'être le premier imitateur et encore moins le plus extravagant; en effet, de nombreuses imitations, qu'elles soient redevables à l'esprit d'Erasme ou de Rabelais ou directement inspirées de Lucien, virent le jour en France depuis le XVlème siècle, se nourrissant toutes du même esprit et faisant parler les morts dans un langage libre et extravagant.

La critique religieuse. Un dialogue intitulé Julius exclusus e coelis fut publié en 1518. Rédigé très certainement entre 1513 - date de la mort du pape Jules II - et la date de sa parution, ce dialogue entre "deux morts célèbres" fut d'abord attribué à Faustus Andrelinus, puis à Erasme - qui l'a nié -, à Jeronimus Balbus, à Coelius Secundus Curio, et plus généralement à Ulric Hutten ${ }^{16}$. Ayant d'abord existé en Latin, ce texte se trouve traduit en Français depuis 1612 - Dialogue entre Saint Pierre et Jules II à la porte du Paradis - et fut plusieurs fois réimprimé jusqu'en 1875, année où Edmond Thion l'a retraduit et publié ${ }^{17}$. Arrivé à la porte du Paradis, le pape-mécène, avec toute sa splendeur et toute sa puissance, au lieu de frapper essaye de l'ouvrir. Mais la réponse, de Saint Pierre certainement, ne se fait pas attendre: "cette porte ne s'ouvre pas avec la clef du coffre fort, je vous en avertis, celle de la Puissance et celle de la Science ne sont pas la même" (1728: 9). Et saisi par un flair insupportable, Saint Pierre, sans savoir encore qui était-ce, ne voulut pas ouvrir et regarda par la lucarne. Voyant alors quelqu'un couvert d'une grande veste où étaient gravées les lettres P.M. ${ }^{18}$, il ne douta pas un seul moment que cela voulait dire "Peste Maudite".

La porte ne s'ouvre pas, mais la conversation a lieu. Parmi toutes les corruptions et ignominies révélées, ce qui choque le plus Saint Pierre, c'est l'hypocrisie de Jules II: II continue, par exemple, d'affirmer qu'il est le neveu de Sixte, alors qu'il sait très bien qu'il est son fils; il se plaint qu'on l'accuse d'être "simoniaque, pédéraste, insensé", et ajoute en guise de justification, pour lui et les autres papes: "ils n'ont point de femmes proprement à eux. Mais pourquoi n'auroient-ils pas des enfants, puisqu'ils ne sont pas eunuques?" (1728: 9). Vraiment ahuri par tant de révélations, Saint Pierre lui demanda si "tous les autres évêques sont faits ainsi", à ce que "Le Génie de Jules" répondit: "la plus part; mais celui-ci est le porte-enseigne de tous" (Ibid: 107). La porte ne s'ouvrit toujours pas, la conversation s'arrêta et Jules II s'éloigna en silence. Voilà une des thématiques présentes dans ce genre de dialogue depuis le tout début de la Réforme protestante jusqu'à la fin du XVIIlème siècle:

\footnotetext{
${ }^{16}$ Cette information se trouve dans le Dictionnaire des ouvrages anonymes, (p.945), de Barbier, apud Domingues, João (2003):148, n.1.

17 - (1875), Julius, dialogue entre Saint Pierre et Jules II à la porte du Paradis, Paris, I. Lisieux, apud Domingues, João, 2003: 148.

${ }^{18}$ Ces lettres désignent le Pape portant le titre "Pontifex Maximus".
} 
contre l'ostentation, le luxe et la débauche des hauts dignitaires de l'Église, qu'ils s'appellent Jules II, Richelieu, Mazarin ou Champvallon, et combien d'autres "bonnets rouges" qui vécurent de la sorte, pour la séduction du pouvoir et pour la gloria mundi.

Toujours dans le domaine de la critique religieuse, mais dans un autre contexte et à plus d'un siècle de distance, un dialogue entre Rabelais et Nostradamus manifeste la même extravagance, surtout lorsque Rabelais raconte que, à peine entré dans les ordres, il décida sur le coup d'abandonner la vie religieuse; dit-il:

aussi ne demeuray-je pas long-tems sous ce froc austere [...] comme je n'avois pas plus le tête faite pour la cucule noire que pour la grize, je la jettai aux orties. (1691: 7)

Et Nostradamus de commenter:

Dieu tira un bon effet d'une méchante cause; car ce fut-là que vous écrivites d'excelens ouvrages sur Hipocrate, et que vous mites en Latin ses aphorismes qui sont un tresor raccourci. (Ibidem: 8)

Mais Nostradamus ne l'épargne pas et lui reproche de, tout chanoine qu'il était, avoir perdu son temps à écrire une "Satire Comique", à ce celui-là répond incontinent:

hé bien, ne vaut-il pas mieux composer un Gargantua que de faire enrager son Evêque, et que de consumer le patrimoine des pauvres en une vie licencieuse et médisante, comme font beaucoup de Chanoines? (Ibidem: 9-10)

La critique politique. Aux côtés de la critique religieuse et de l'expression d'un esprit rebelle, voire irréligieux, qui plonge ses racines en plein seizième siècle, la critique politique fait aussi son apparition. Dans l'ouverture d'un de ces dialogues, voilà ce que dit Mahomet à Colbert lorsqu'il débarque en l'autre monde:

Soyez le bien-venu, Monsieur Colbert, (...) A tous momens on parle icy de vous, si nos Maîtres mettent au cul de quelques impudiques des flambeaux ardens, ils crient aussi-tôt: Flambeaux de la Manefacture de Colbert. S'ils endossent à des Soldats brigands des Tuniques brûlantes, ils ajoûtent: Ce sont Buffles de la Manefacture de Colbert. S'ils coiffent quelques Coquettes d'une maniere qui represente la mode; ils soûtiennent, Que ces coiffes sont du point de la Manefacture de Colbert; et même s'ils ténaillent quelques malheureux, ils veulent persuader qu'ils font une levée et une taxe de l'invention de Colbert. (1683: 1) 
Colbert n'a pas frappé à la porte du Paradis; au contraire, lui et son illustre amphitryon Mahomet, ils sont entrés directement en enfer: à ce sujet, le titre du dialogue ainsi que l'environnement, bien que laconiquement décrit, ne laissent aucun doute au lecteur. Durant la fête de réception du nouveau-venu, on voit que tous les personnages avaient beaucoup de caractéristiques en commun, et il n'est pas difficile de deviner lesquelles; mais ce qui transforme le dialogue en vraie tragicomédie humaine est bien le langage: tout est nommé par son nom - vices et crimes compris - sans aucune retenue.

Un autre texte du même moule est l'Entretien d'Auranphile avec Veneredelphe. Le personnage principal est un "maltotier" qui, comme son nom l'indique, "aimait les richesses"; mais il n'aimait pas moins les actrices de l'Opéra avec qui il dépensait largement sa fortune. Dans le dialogue, il raconte à son ancienne amie Veneredelphe - "sœur de Vénus", c'est ce que veut dire son nom - qu'ici aux enfers il a été battu par Pluton en personne et persécuté par toute une troupe de "Galfretiers tous noirs, ayant des cornes à la tête, [qui l'] auroient tué" (1708:6-7), malgré l'absurde de l'expression pour la circonstance. La comédienne ne se plaint pas de son sort mais le sort du "maltotier" ne la surprend pas non plus; elle le trouve fort différent de ce qu'il était en l'autre monde et observe simplement: "je me souviens", ditelle, "lorsque vous étiez dans l'autre monde, vous aviez un embonpoint, et presentement je vous trouve sec, aride et fort noir" (Ibid.: 11). Comique, satirique et grotesque à la fois, car on se rie des crimes comme des défauts physiques des condamnés, y compris de la laideur, de l'âme comme du corps. C'est bien d'extravagance rabelaisienne qu'il s'agit, avec tout son langage faussement imbécile. Ainsi donc, si le lecteur prend l'auteur pour un imbécile et n'y voit que des ordures, il rira, certes; mais si au contraire il y voit quelque finesse d'esprit et comprend l'étendue de la chose, alors il rira encore plus.

Toujours en enfer, lorsque le sujet de la guerre est abordé, le langage grotesque est lui-même surpassé par les descriptions macabres, puisqu'il s'agit de rendre compte de la plus grande extravagance des humains sur terre:

II vint icy de cette bataille une infinite de Circoncis $^{19}$. Je leur demanday quelle nouvelle, ils me répondirent, triste pour nous, force coups en l'autre monde, et l'Enfer en celuy-cy. L'un avoit la teste fenduë jusqu'au menton, l'autre portoit ses entrailles en ses mains; tel sautoit sur un pied ayant perdu l'autre; tel autre estoit devenu tout de porfil, ayant la moitié de la face emportée. II y en eut mesme un qui me fit rire, tout désolé que j'étois, car l'Epée de son ennemy luy estant entrée par l'anus, luy sortoit par la bouche, et sembloit un Curedent, ce

\footnotetext{
${ }^{19}$ II s'agit ici d'un massacre perpétré par des chrétiens contre des musulmans qui, comme les juifs, avaient aussi la circoncision dans leurs pratiques religieuses.
} 
qu'il disoit estre l'effet du zele d'un Apotiquaire Chrestien. Enfin j'appris d'eux l'infortune de nostre parti, et l'appris avec le desespoir d'un Damné.

(1683: 11-12)

Critique et parodie de l'Histoire ou comédie de mœurs. Parfois, certains dialogues font la parodie de l'histoire ou ressemblent à des comédies de mœurs extravagantes. Prenons comme exemple l'Entretien de Scarron et de Molière (1690). Le dialogue commence avec une grêle de coups de la part de Caron persécutant Molière dans les rues de Paris. Le Passeur de la barque infernale, "qui crioit par tous les cartiers, aux badauts, aux badauts; et qui chassait, à coups d'aviron tout ce qu'il rencontrait de Parisiens, pour leur faire repasser le Stix" (1690:4), était en colère contre Molière parce qu'il croyait que, comme tous les parisiens, il avait annoncé et fêté la mort du Prince d'Orange, alors qu'il était encore vivant. Cette scène qui fait allusion à l'Histoire de France, certainement au moment de l'alliance de l'empereur germanique avec le duc de Lorraine, le roi d'Espagne et le Prince d'Orange, le roi d'Angleterre contre Louis XIV, en 1673, fut d'abord le prétexte d'une extravagance pleine d'imagination littéraire: Scarron fut obligé d'inventer un stratagème poétique "pour se mettre à couvert des bacules de ce Nautonier"; au moment où Caron lève la rame pour le rouer de coups "contre les fesses", il improvise ce quatrain qui fut son salut:

\author{
Les Badauts sont extravagans \\ De mettre en terre les vivans \\ Vive, vive le Roi Guillaume \\ II rentre vif en son Roiaume. (Ibidem: 5)
}

Molière, lui, n'a pas eu besoin de se justifier parce que les persécuteurs ont cru que sa comédie du Malade imaginaire ne faisait que se moquer du faux bruit du "mort imaginaire".

Puis la comédie littéraire se transforme subtilement en parodie de l'histoire lorsqu'on raconte les "fourberies" que Scapin a réalisées depuis qu'il est arrivé aux Champs-Élysées: en fait, il commença par soûler Caron en personne et lui couper la bourse; puis il a rasé le Duc d'Alve et en a fait un mignon; il a vendu une perruque au Duc d'Orange, le fondateur de la République de Hollande, et une paire de lunettes au roi de Suède qui avait la vue courte; il a offert un coussin à la reine Christine "pour faire l'oraison de quiétisme à la Molinos", un plan de Pavie à François I., et un chapeau noir au cardinal Mazarin, qui avait accédé au cardinalat sans jamais avoir été prêtre. II a vendu une tabatière au Prince de Condé et il a 
offert à Louis XI un perroquet qui répétait sans cesse "Perone, Perone"20. Et pour comble de "scapinade" extravagante - mais aussi comble de liberté d'expression pour l'auteur de ce dialogue -, "le petit coquin" s'est adressé à Scarron et lui a offert "d'une main une jate pour y placer [son] cul, et de l'autre le portrait de la Marquise de Maintenon" (Ibidem: 22-24), veuve du poète, mais qui plus est, épouse du roi Louis XIV au moment même de cette publication clandestine et anonyme! Le texte se termine, enfin, par une scène du monde à l'envers où les vrais "bourgeois", "malades imaginaires", "cocus imaginaires" ou "femmes savantes", "misanthropes" et "fâcheux" parisiens, loin de se fâcher contre Molière qui les a tant fouettés dans ses comédies à Paris, acceptent tout joyeux de représenter leurs propres rôles dans des impromptus aux Champs-Élysées.

L'extravagance du langage. Souvent, indépendamment du sujet abordé, ce qui émerge tout de suite dans ce genre de dialogues est la liberté de langage que s'arrogent les personnages, c'est-à-dire les morts. Ils n'hésitent pas non plus à faire appel aux domaines les plus provocateurs, les moins édifiants et les moins propres de la vie des hommes. Et plus on avance dans le XVIIlème siècle, plus cette extravagance est fréquente et explicite. C'est ce qui arrive dans de nombreux dialogues de morts qui composent les Entretiens des Ombres de Jungerman, publiés en 1722. Par exemple, dans un dialogue entre Molière et Sixte V, celui-ci parle des chanteurs de musique religieuse en disant que ce sont "des chapons dont on a féminisé la voix aux dépens de la postérité" (Jungerman, 1722: t.1, 140). Non content de ce langage, le Pape cite, sans aucune contrainte, des vers de certains "Poetereaux" italiens comme Carlo Matera qui fut envoyé aux galères pour avoir terminé son poème au sujet d'une dame de la façon suivante: "in somma questa Dama é gran Puttana". S'il arrive à ce pape de citer de grands personnages de l'Église, tel le cardinal Farnese, c'est pour citer des observations comme celle qui apparemment resta célèbre à propos de l'élection papale d'un vieux cardinal: "ils ont cru [...] faire un Pape qui seroit un coyon, mais je prévois qu'ils se sont donné un Maître qui les coyonnera tous" (Ibidem: 170). Dans d'autres dialogues, il y a des histoires de nudités féminines honteuses, d'actes, de gestes et d'odeurs les moins dignes, tous racontés de manière explicite et sans pudeur. Loin du langage de la cour, loin aussi des fers de l'orthodoxie morale et langagière auxquels l'académie souhaitait attacher toute la gent cultivée, ces textes se plaisent dans la provocation d'autant plus efficace qu'elle sort de la bouche de morts qui furent des grands de ce monde. Si le sujet est le comportement féminin, c'est toute une pléiade de termes et d'expressions grivoises qui surgissent, soit pour caractériser les déviations du comportement féminin, soit pour reproduire la fausse ignorance que les femmes utilisent pour se justifier auprès des maris bernés. Quand enfin, dans la seconde moitié du siècle, il n'y a plus de

\footnotetext{
${ }^{20}$ Louis XI fut obligé de signer un humiliant traité en 1468, devant Charles le Téméraire, précisément à Péronne, région de la Somme.
} 
secret sur la vie privée de Louis XV et de son cercle, "le cercle des roués", le langage des morts n'a plus aucune retenue: aux enfers, Louis XV en personne pleure parce qu'il n'a plus son "Parc-aux-cerfs", ni sa chère Du Barry qu'il aurait fait reine, car, dit-il, "mon bis-yeul en a bien fait autant avec la prude et dégoutante veuve du bossu, cul-de-jatte, Poëte Scaron" (1784: 86). Le même roi crie, éperdu, quand il apprend que le Duc d'Aiguillon avait "fouillé sa couche": "Ces coups d'aiguillon me roulent encore dans ma tête. J'ai donc été COCU, COCU [...]!" (Ibidem: 37). Enfin, des morts anonymes dénoncent vertement les crimes des ministres comme les vices des rois sans laisser d'avoir recours aux gros mots, à la coprolalie et au langage érotique grossier. Si la naissance de la presse périodique montre qu'on a enfin compris l'importance de l'opinion publique, ce genre de "littérature marginale", souvent anonyme et clandestine, est à l'image - elle en est l'image - du mécontentement d'un bon pourcentage de la société française en marche vers la fin de l'Ancien régime et la Révolution.

Dans la structure même des dialogues, dans le choix des paires qui conversent comme dans la façon dont les échanges se déroulent, l'extravagance se constitue en maîtresse du discours entre les morts et le jeu du paradoxe devient la condition indispensable de la réflexion. Son absurdité donne le ton et, dès lors, ce n'est plus la logique qui commande: la logique du bon sens s'étiole, perd son sens, et demande à ce que l'on refasse, dès le début, un nouveau sens de la réalité; car tout est remis en cause et bouleversé dans l'autre monde qui est vraiment autre, un monde à l'envers.

Les N.d.m. de Fontenelle et quelques autres petits chefs-d'œuvre du genre font revivre toute l'extravagance de l'original grec de Lucien de Samosate. Par ailleurs, ce sousgenre littéraire, souvent réduit au statut de littérature de colportage, découvre, surtout au XVIlème siècle, la liberté d'expression qui lui permet d'être impudique, manifestant un libertinage érudit qui ne cessera d'augmenter tout au long du XVIIIème. Mais toute cette extravagance est au bout du compte un masque, une stratégie, un jeu du langage pour séduire le lecteur, qu'il conduit, par l'éclatement même du dialogue, droit à la critique sérieuse. Cette critique concerne la société, la politique et la religion, ou alors elle s'immerge dans le sérieux débat d'idées, apanage de ce siècle en France.

Nourri des extravagances de toute sorte, le "dialogue des morts" - mis à part les grands textes de Fontenelle, Jungerman et quelques autres - est un sous-genre de littérature typiquement clandestine et de colportage, persécutée par la Censure, voisinant donc avec la littérature pornographique, les almanachs, les libellés politiques et les feuilles volantes de toute sorte. Et pourtant, la diversité de textes produits, le nombre d'auteurs 
concernés et la richesse des sujets qu'il comporte ne laissent aucun doute sur l'importance de l'analyse d'exemples de cette espèce dans l'étude de l'histoire et de la culture de cette époque. Quoi qu'il en soit, la myriade d'observations qu'ils présentent, secondée par la qualité de certaines critiques, laisse percevoir l'importance de ces "révélations" libres et sans contraintes, lucides et objectives pour la compréhension des réalisations humaines de l'époque, surtout en ce qui concerne tout ce qui était fait et dit, pensé et écrit, en public ou en privé, à défaut des institutions. On y trouve l'histoire vue par le bas-fond, mais un bas-fond très proche des "esprits forts", sinon coïncidant même avec le "libertinage érudit", contestataire et libertin, et nullement ignorant.

Toutes extravagantes que puissent paraître ces "descentes littéraires aux enfers", elles se transforment, au fur et à mesure, par le regard supérieur et non affecté des morts, en réflexion profonde sur ce qu'est l'être humain sans ambigüité et nous révèlent sa condition existentielle sans illusion. 


\section{Bibliographie}

Corpus:

Ablancourt, Nicolas Perrot, Sieur de (1654) Lucien de Samosate, de la traduction de Nicolas Perrot d'Ablancourt, 2 vol.s, A. Courbé, Paris.

ANONYME $(1518,1728)$. Dialogue entre Saint Pierre et Jules Il à la porte du Paradis.

(1683). Entretien dans le royaume des Ténèbres, sur les affaires du Temps, entre Mahomet et Mr. Colbert, cy-devant ministre de France.

(1690). Entretien de Scarron et de Molière.

(1691). Entretien de Rabelais et de Nostradamus.

(1708). Entretien d'Auranphile avec Veneredelphe, dans les Champs Elisées, sur les affaires de ce monde.

Vayer).

(1716), Cinq Dialogues faits à l'imitation des Anciens par Oratius Tubero (La Mothe Le

(1784). Les entretiens de l'autre monde sur ce qui se passe en celui-ci.

FONTENELLE, Bernard Bouvier de $(1683,1971)$. Nouveaux dialogues des morts, Édition critique avec une introduction et des notes par Jean Dagen, Librairie M: Didier, Paris.

JUNGERMAN, Valentin $(1722$ - 23). Entretiens des Ombres aux Champs Elysées sur divers sujets d'histoire, de politique et de morale (3 vols.), chez Herman Vitwerf, Amsterdam.

VoltAIRE, François Marie Arouet de $(1765,1961)$. "Conversation de Lucien d'Érasme et de Rabelais dans les Champs-Elysées", in Dialogues satiriques et philosophiques, dialogue V, dans Nouveaux mélanges, Paris.

\section{Études:}

CARRE, Jean-Raoul (1932). La philosophie de Fontenelle ou le sourire de la raison, F. Alcan, Paris.

ChARLES-DAUBert, Françoise (1998), Les libertins érudits en France au XVIle siècle, éd. PUF, Paris.

DOMINGUES, João (2003). O diálogo dos mortos, de d'Ablancourt a Voltaire, FLUC, Coimbra (tese policop.).

(1999). "O pensamento de Fontenelle nos Nouveaux dialogues des morts", in Da natureza ao sagrado, vol. I, Fundação, Eng. António de Almeida, Porto: 289 - 312.

FAYOL, A. (1961). Fontenelle, Nouvelle éd. Debresse, Paris.

MOREAU, Isabelle (2007). "Guérir du sot”. Les stratégies d'écriture des libertins à l'âge classique, Honoré Champion, Paris.

NIDERST, Alain (1991). Fontenelle, éd. Plon, Paris.

PINTARD, René (1943, 2000). Le Libertinage érudit dans la première moitié du XVIle siècle, Nouvelle édition augmentée, éd. Slatkine, Paris. 